\title{
L'apport Des Marchés De Capitaux Au Financement Des PME Marocaines
}

\author{
M. Mohamed Oudgou \\ M. Mohamed Zeamari \\ Laboratoire d'Études et de Recherches Économiques et Sociales (LERES). \\ Faculté des sciences juridiques, économiques et sociales. \\ Université Moulay Ismaïl, Meknès, Maroc
}

Doi: 10.19044/esj.2018.v14n7p350 URL:http://dx.doi.org/10.19044/esj.2018.v14n7p350

\begin{abstract}
Moroccan SMEs do not use generally capital market due to rigidity of the conditions. Moroccan authorities have introduced the measurements to deal with this problem and to encourage SMEs to access in the capital market since 2000. However, these measures were less efficient because they are multiple reason, but indeed, it still attributed to the financial and institutional conditions of access to capital market. The article target is to analyze capital market reforms and its contribution to financing Moroccan SMEs. In order to help us to determine the constraints of access the SMEs to direct finance. In fact, to achieve the target we need to adopt a methodology based on the analysis of secondary statistics and official reports of the main actors of the capital markets in Morocco.
\end{abstract}

Keywords: Finance, Capital market, SMEs, access to finance.

\section{Résumé}

Dans plusieurs études sur le financement des PME marocaines, il est constaté que cette catégorie d'entreprises ne recourent que très faiblement aux marchés de capitaux. Afin de faire face à cette problématique, les autorités marocaines ont mis en place une panoplie de mesures depuis le début des années 2000. Malgré les efforts déployés, le troisième compartiment de la bourse de Casablanca ne comporte qu'un nombre très limité de PME. Les causes sont, en effet, multiples mais, sont toujours imputées aux conditions financières et institutionnelles d'accès aux marchés de capitaux. L'objectif de cet article est d'analyser l'ampleur des réformes des marchés de capitaux et leurs apports au financement des PME marocaines. Pour atteindre cet objectif, nous adoptons une méthodologie fondée sur l'analyse des statistiques 
secondaires et des rapports officiels des principaux acteurs des marchés de capitaux au Maroc.

Mots-clés : Finance, Marchés de capitaux, PME, accès au financement.

\section{Introduction}

L'aide en faveur de la promotion des PME est née à la fin des années 1970 aux Etats-Unis. Ceci, constitue une «distorsion au régime de la concurrence que les pouvoirs publics américains justifient par plusieurs raisons. La première repose sur le processus de destruction-créatrice : il convient d'aider les PME à devenir les champions de demain. La deuxième raison réside dans l'aptitude de ces entreprises à créer des emplois... » (Levratto, 2009). La politique publique américaine en faveur des PME repose sur la distribution des crédits à des taux privilégiés et la garantie ${ }^{1}$. La GrandeBretagne a suivi le modèle américain, et met en place à la fin des années 1970 un système de garantie des crédits dédié aux PME complété par un plan d'expansion et la création d'un marché des capitaux réservé aux entreprises non cotées.

La prise en compte des PME marocaines par le gouvernement remonte au début des années 2000. A partir de là, et compte tenu de leur importance dans le tissu économique national et leur sous-capitalisation, que des politiques d'appui soient lancées en leur faveur. Ce n'est qu'à partir de 2002 qu'un renforcement de ces politiques est apparu suite à la promulgation de la charte des PME. Cette charte a défini, pour la première fois, la PME marocaine et a mis l'accent sur une dizaine de politiques prioritaires notamment, la création de l'ANPME', l'allégement et la simplification des règles juridiques dans les domaines financiers, fiscales et fonciers, la promotion de l'innovation via la création des fonds d'investissement en capital, la création des sociétés régionales de financement, etc. Parallèlement, des actions d'envergure ont été lancées depuis 2003 pour la sensibilisation des TPME sur les actions des politiques publiques en leur faveur, sur les conditions de leur financement, sur la législation fiscale et sociale, etc. En outre, le soutien public au financement des PME marocaines est renforcé par la refonte du système de garantie national en 2009. Dès lors, L'Etat s'appuie exclusivement sur les fonds de garanties la

${ }^{1}$ Les politiques publiques américaines en faveur des PME reposent sur la loi fondatrice appelée le Small Business Act (SMA). Ce dernier a été créé par Small Business Administration (SBA) et voté le 30 juillet 1953 par le congrès américain. La SBA se charge aujourd'hui de toutes les aides aux PME, qu'il s'agisse du financement, du conseil, de la formation, de l'accès aux marchés publics ou du lobbying en faveur des PME.

${ }^{2}$ En conséquence de la création de l'Agence Nationale pour la Promotion de la PME (ANPME), l'Office pour le Développement Industriel (ODI) a été dessous. ODI est une institution publique ayant eu pour mission de développer le tissu industriel marocain. Actuellement cette agence est nommée Maroc-PME. 
Caisse Centrale de Garantie (CCG) pour supporter une partie du risque des PME couru par les établissements bancaires.

Dans le même ordre, l'Etat a mis en place plusieurs actions afin de faciliter l'accès des PME aux marchés de capitaux. Les marchés boursiers procurent aux PME des ressources financières directes pour financer leur développement et leur assure une visibilité (CDVM, 2011). A la fin des années 70, des pays européens ont reproduit l'exemple des Etats-Unis des Small Caps du NASDAQ et ont créé des compartiments boursiers dédiés spécialement aux PME afin de renforcer leurs fonds propres. Le Maroc reproduit l'expérience européenne en créant un troisième compartiment réservé aux entreprises moyennes. En dépit des réformes des marchés des capitaux (marché monétaire et marché financier) en 1993, le financement de l'économie en général et des PME en particulier demeure limité. Les réformes introduites ont été manifestées par l'inefficacité de la politique de change et celle de l'encadrement de crédit. A cet effet, les autorités marocaines ont affiché une volonté ambitieuse de faire de Casablanca une place financière via la promulgation de la loi de 21 septembre portant réforme des marchés des capitaux. Cependant, ces efforts demeurent vains en raison de la distorsion entre les exigences d'accès à ces marchés et les caractéristiques des PME marocaines. Jusqu'à quel point les efforts entrepris ont permis aux PME de se financer via le marché des capitaux marocain?

Dans le présent article, nous nous focalisons notre attention sur le financement direct des PME marocaines par le marché de capitaux et les actions menées dans ce cadre pour inciter ces entreprises à y accéder davantage.

\section{Les réformes et la structure du marché des capitaux}

La volonté des réformes des marchés des capitaux s'est portée à la fois sur le marché financier que sur le marché monétaire. Ces réformes visaient la modernisation du système financier marocain afin d'accroître son efficacité en matière de financement du développement du pays et d'accroitre son attractivité à l'échelle mondiale. Le dynamisme des réformes s'est couronné par la création de Casablanca Finance City qui se place comme hub économique et financier de dimension régionale.

\subsection{La modernisation du marché monétaire}

Le marché monétaire est le marché de capitaux à court et moyen terme. Ce marché permet à ses opérateurs de désintermédier leurs placements et financement à court et moyen terme. Réservé initialement aux opérations bancaires et réorganisé entre 1983 et 1988 en deux sous marché : le marché interbancaire et le marché monétaire élargi. La réforme de 1993 a concerné deux volets : le premier est relatif à la dynamisation du marché des 
adjudications des bons de trésor et le deuxième est relatif à la création des titres de créances négociables.

\subsubsection{Le marché interbancaire (marché monétaire)}

Ce marché permet la rencontre de l'offre et de la demande de liquidités bancaires entre les établissements de crédit (les banques, les sociétés de financement et la Bank Al-Maghreb) et les sociétés d'investissements. Il permet à ces établissements de s'approvisionner en liquidités à court terme. Ce marché soumis au contrôle de Bank Al-Maghreb qui par ses interventions contrôle la masse monétaire et les crises du système bancaire. Les interventions de Bank Al-Maghreb peuvent être effectuées via les billets de trésorerie et/ou les bons de trésor $^{3}$ afin d'injecter ou retirer la liquidité moyennant un taux d'intérêts.

Tableau 1 : L'évolution du taux d'intérêt sur le marché interbancaire (\%)

\begin{tabular}{lllllllllllllllll}
\hline Année & 2000 & 2001 & 2002 & 2003 & 2004 & 2005 & 2006 & 2007 & 2008 & 2009 & 2010 & 2011 & 2012 & 2013 & 2014 & 2015 \\
\hline Taux & 5,35 & 3,23 & 2,94 & 3,16 & 2,39 & 2,40 & 2,58 & 3,29 & 3,37 & 3,35 & 3,29 & 3,28 & 3,19 & 3,06 & 2,94 & 2,51 \\
\hline
\end{tabular}

Source : Elaboré par nous-mêmes du tableau de bord des indicateurs macroéconomiques(DEPF, 2016).

Le tableau ci-dessus nous montre l'évolution du taux d'intérêt sur le marché interbancaire. Le taux d'intérêt s'est évolué entre 2005 et 2009 de 2,4\% à 3,35\%, ce qui renseigne sur l'augmentation de la demande de la liquidité de Bank Al-Maghreb par les établissements de crédit. En effet, en 2008 où la crise financière s'est accrue, la demande sur la liquidité s'est accentuée et le taux interbancaire atteint son maximum. Entre 2010 et 2015 le taux baisse pour se situer à $2,51 \%$, ce qui traduit une baisse de la demande de la monnaie de la banque centrale. Le taux moyen annuel entre 2000 et 2007 est de 3,2\% et de $3,1 \%$ entre 2008 et 2015.

\subsubsection{Le marché des titres de créances négociables (TCN)}

Le marché des titres de créances négociables (TCN) appelé marché monétaire élargie. Le dispositif réglementaire de la réforme de ce marché a été mis en place en janvier 1996. Avant cette réforme, ce marché n'était accessible qu'aux établissements bancaires et aux Organismes Financiers Spécialisées (OFS). L'adoption de la loi relative aux TCN a élargi le marché aux Trésor, aux sociétés de financement et aux entreprises. A côté des bons de trésor (existant depuis 1989) de nouveaux titres ont été créés et sont de quatre

\footnotetext{
${ }^{3}$ Ces opérations s'appelle la mise en pension et consiste en un transfert simultané, entre deux parties, de titres contre une certaine somme d'argent, avec l'engagement des deux parties de procéder au transfert inverse à une date ultérieure.
} 
catégories actuellement: les bons de trésor émis par l'Etat (le trésor) ${ }^{4}$, les billets de trésorerie émis par les entreprises privées ou publiques non financières ${ }^{5}$, les certificats de dépôts émis par les banques ${ }^{6}$ et les bons des sociétés de financement ${ }^{7}$. Ces titres sont émis au gré de l'émetteur pour financer ses besoins. La loi autorise tous les investisseurs à accéder à ce marché, y compris les personnes physiques via les Organismes de Placement Collectif en Valeurs Mobilières (OPCVM). Il y a donc une libéralisation du marché et une augmentation de la concurrence entre les intervenants. De ce fait, les emprunteurs peuvent se financer ou placer la liquidité sur un marché ouvert à des meilleures conditions.

Graphe 1 : Evolution de l'encours des Titres de Créances Négociables (TCN) (en millions de dirhams)

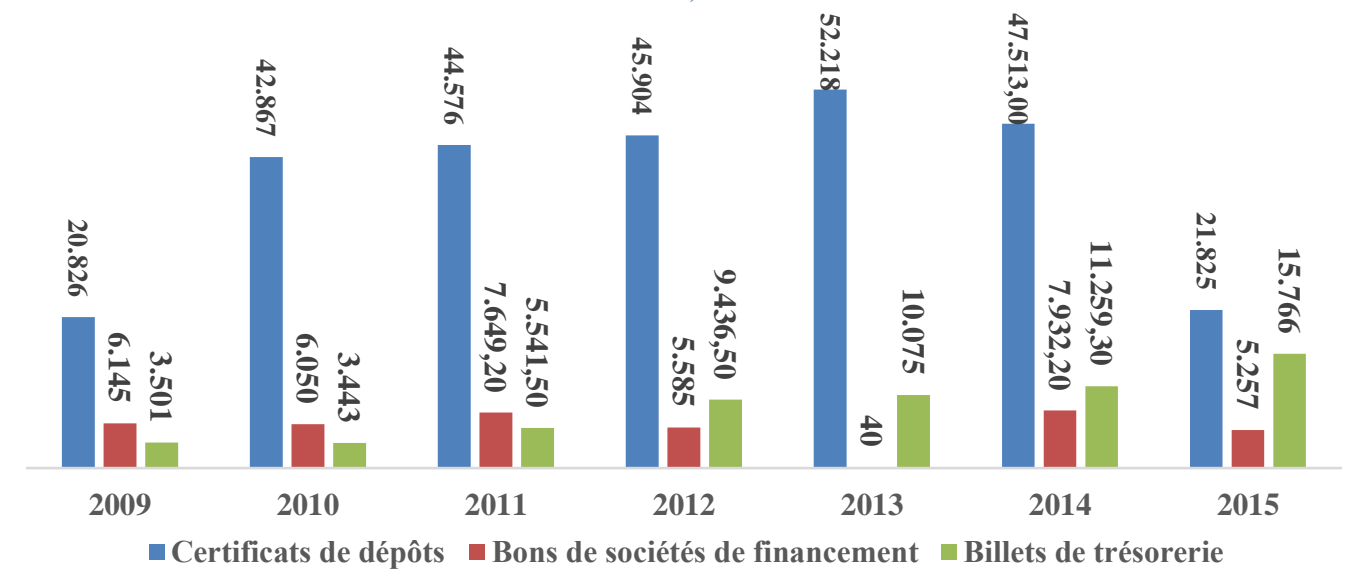

Source : Elaboré par nous-mêmes à partir des rapports annuels de (CDVM, 2014 et 2015)

Les émissions des TCN n'ont cessé de se progresser depuis la première opération en octobre 1996. A cette période les Certificats de dépôts représentaient 1626 millions de dirhams et les Billets de Trésorerie 148 millions de dirhams, alors qu'aucune opération d'émission des Bons de Sociétés de financement n'a été effectué. Le graphe ci-dessus nous montre la dominance des Certificats de Dépôts avec en moyenne $70 \%$ des opérations des TCN, non seulement sur la période de 2009 à 2015, mais dès leurs créations 1996. Les banques sont donc les principaux émetteurs sur le marché des TCN.

${ }^{4}$ Les Bons de trésor ont une durée qui varie de court terme (12, 26 et 52 semaines), moyen terme ( 2 à 5 ans) et de long terme (12,15 et 20 ans) remboursables in fine. Le montant est fixé par voie réglementaire.

${ }^{5}$ Billets de trésorerie : émis par les sociétés ayant au moins 5 ans d'existence, trois bilans certifiés. Leur achat s'effectue à travers les OPCVM. Le montant du billet est de $250000 \mathrm{Dh}$ minimum et la durée de 10 jours à 1 an.

${ }^{6}$ Certificats de dépôts : montant minimum de $50 \mathrm{Mdh}$ et d'une durée de 10 jours à 7 ans.

${ }^{7}$ Les bons des sociétés de financement : émis par les sociétés de financement et dont la durée est de 2 à 7 ans. 
Ces titres permettent de financer les besoins de trésorerie à des taux relativement faibles. De plus, ces titres répondent aux besoins spécifiques de son émetteur.

L'évolution des émissions des instruments de financement sur le marché monétaire prouve la réussite des réformes engagées par les autorités marocaines. Ces réformes ont concerné aussi le marché financier.

\subsection{La réforme de la bourse des valeurs mobilières et la création de Casablanca Finance City}

Le marché financier marocain c'est le marché des valeurs mobilières (actions et obligations), appelé marché des capitaux à long terme. Ce marché (la bourse de Casablanca), a connu une série des réformes depuis sa création en 1929. La première réforme introduite en 1948 a attribué à la Bouse de Casablanca le statut de «personnalité morale ». La deuxième, en 1967, a permis de la réorganiser à la fois techniquement et juridiquement et de la définir en tant que « établissement public » (BVM, 2012). Une nouvelle phase des réformes a commencé depuis 1993 pour doter l'économie marocaine d'un système financier moderne, efficace et dynamique dans la mobilisation de l'épargne vers le circuit économique. Les réformes ainsi introduites lui ont permis d'augmenter le nombre des entreprises, d'accroitre le volume des transactions, d'améliorer son attractivité mondiale et de se positionner comme leader en continent Africain ( $3^{\text {ème }}$ ).

Dans ce qui suit, nous présentons les grandes lignes des réformes de la Bourse de Casablanca, avant d'analyser l'évolution de ses principaux indicateurs et sa contribution au financement de l'économie marocaine en général.

\subsubsection{Synthèse des réformes du marché financier}

La réforme et la modernisation de la Bourse de Casablanca s'est attachée à la création du Conseil Déontologique des Valeurs Mobilières (CDVM) en juillet 1994, à la création de la société gestionnaire : Société de la Bourse des Valeurs Mobilières en août 1995, à la création des Organismes de Placement Collectifs en Valeurs Mobilières (OPCVM) en novembre 1995, la création du Dépositaire Centrale Maroclear en décembre 1996 et à l'ouverture de son capital en novembre 2015.

La réforme s'est attachée en premier lieu à l'instauration d'une entité de contrôle, en l'occurrence le Conseil Déontologique des Valeurs Mobilières (CDVM). Le CDVM, est un établissement public doté de la personnalité morale et de l'autonomie financière. Sa principale fonction est d'assurer la transparence et le bon fonctionnement du marché boursier à travers le contrôle et le respect des dispositions légales et réglementaires les régissant. Il s'agit de protéger l'épargne, les investisseurs et de sanctionner le non-respect des règles 
par les opérateurs (sociétés de bourse, entreprises, investisseurs). En 2004, 2006 et 2007, des réformes ont été introduites pour renforcer le pouvoir de contrôle du CDVM et d'améliorer le système de sécurité des transactions. Cet établissement est devenu en Novembre 2016 l'Autorité Marocaine du Marché des Capitaux (AMMC) avec l'intervention dans le contrôle de la stabilité financière du système financier marocain et avec plus d'indépendance dans l'exercice de ses missions.

S'agissant de la création des sociétés de bourse ${ }^{8}$ (remplaçant les agents chargés de change), à fin de 2016 sont de $17^{9}$. La réforme de 1993 a attribué à ces sociétés le monopole de l'intermédiation boursière. Elles sont donc devenues habilitées à exécuter les transactions sur les valeurs mobilières (actions et obligations) pour le compte de leurs clients. Ces sociétés sont autorisées également en vertu d'un mandat à effectuer des opérations de placement des titres émis par des personnes morales et en assurer la garde et la gestion, le conseil de la clientèle, l'assistance lors des introductions en bourse et les opérations d'ingénierie financière.

Afin de canaliser l'épargne vers la Bourse de Casablanca, les réformes sont couronnées par la création des OPCVM en novembre 1995. Les OPCVM sont des organismes financiers dont les fonds investis sont placés en actions, obligations et en liquidités ; offrant aux porteurs de part ou aux actionnaires une parfaite liquidité. Les amendements apporté au cadre juridique des OPCV en 2004 ont permis de distinguer, juridiquement entre les Sociétés d'Investissement à Capital Variable (SICAV) et les Fonds Collectifs de Placement $(\mathrm{FCP})^{10}$. Selon la stratégie d'investissement, la loi distingue entre les OPCVM actions, obligations, monétaires, contractuelles et diversifiées. D'autres amendements sont entrés en vigueur en 2006 afin d'assurer une meilleure transparence et la protection des épargnants.

Le processus des réformes de la Bourse a coïncidé avec l'informatisation du système de la cotation, la dématérialisation des valeurs mobilières et la création d'un dépositaire central : Maroclear en 1996, suite à la promulgation de la loi $\mathrm{n}^{\circ} 35-96$. Maroclear est une société anonyme (SA) sous le contrôle du Ministère des Finances et son capital et détenu par: la

${ }^{8}$ Le capital lors de la constitution ne peut être inférieur à $1500000 \mathrm{dh}$ et ne peut être inférieur à 5000000 dhpour celles qui veulent agir pour leur propre compte. Ainsi, ne peuvent exercer qu'après agrément du ministère chargé des finances sur avis de l'AMMC.

${ }^{9}$ Les 17 sociétés de bourse sont: Alma Finance Groupe, Artbourse, Atlas Capital Bourse, Attijari Intermédiation, BMCE Capital Bourse, BMCI Bourse, Capital Trust Securities, CDG Capital Bourse, CFG Marches, Crédit du Maroc Capital, ICF Al Wassit, MENA.C.P, MSIN, Sogecapital Bourse, Uplines Securities, Valoris Securities, Wafabourse.

${ }^{10}$ Les SICAV sont des sociétés anonymes à capital de 50 Millions de dirhams minimum et ont pour objet la gestion d'un portefeuille de valeurs mobilière et de liquidité, alors que les FCP représentent une copropriété des valeurs mobilières et de liquidités. Les FCP n'ont pas le statut de personne moral. 
Bourse (5\%), la CDG (10\%), les compagnes d'assurances (15\%), les banques (25\%), Bank Al-Maghreb (20\%) et par l'Etat (25\%). Une telle répartition offre une représentation de l'ensemble des intervenants à la Bourse de Casablanca et elle compte actuellement 178 affiliés (Maroclear, 2015). Maroclear a pour mission d'assurer la codification des valeurs mobilières, la conservation et l'administration des valeurs mobilières pour le compte de ses affiliés, de gérer les comptes des valeurs mobilières ouverts au nom de ses affiliés et leurs faciliter l'exercice des droits attachés aux valeurs mobilières et à l'encaissement des produits qu'ils génèrent ${ }^{11}$.

D'autres réformes d'ordres techniques ont été entamées en parallèle afin de moderniser l'organisation du marché boursier. Il s'agit notamment de la mise en place d'un système de cotation électronique en 1998 et sa modernisation en 2008 afin d'assurer une rapidité d'exécution des ordres de bourses avec transparence et sécurité. L'adoption de la capitalisation flottante et le lancement en 2002 de nouveaux indices de capitalisation (MASI et MADEX). Le remplacement du Marché Officiel et du Marché des Cessions Directes par le marché Central et par le Marché de Blocs. La création du troisième compartiment dédié aux PME.

Selon le Dahir $n^{\circ} 1-211-93$ relatif à la Bourse des Valeurs de Casablanca, le capital de la bourse est détenu à parts égales par les sociétés de la bourse. Ce modèle «mutualiste» atteint ses limites au niveau de l'unicité du corps de gouvernance ce qui limite l'évolution du marché (Ministère de l'Economie et des Finances, 2016). Afin d'accompagner le financement du développement économique du pays, l'ouverture du capital de la Bourse est devenue une nécessité. Le projet d'ouverture de la bourse fait référence à l'amendement du Dahir susmentionné suite à la signature en novembre 2015 d'un mémorandum d'entente entre l'Etat, l'AMMC, les actionnaires actuels de la Bourse de Casablanca et ses futurs actionnaires. De ce fait, l'actionnariat de la Bourse sera réparti entre les banques (39\%), la CDG (25\%), les sociétés de bourses indépendantes (20\%), les sociétés d'assurances (11\%) et Casablanca Finance City Authority $(5 \%)^{12}$. La diversification des actionnaires permettra à la Bourse de passer à un nouveau pallier de croissance et de s'ouvrir sur le continent Africain. Le mémorandum d'entente a prévu également la transformation de la Société Gestionnaire de la Bourse en une Société Holding.

Le processus de modernisation du secteur financier marocain a été couronné par l'adoption de la loi relative à la création de Casablanca Finance

${ }^{11}$ Dahir n¹-96-246 du 29 Chaabane 1417 (9 janvier 1997) portant promulgation de la loi $\mathrm{n}^{\circ} 35-96$ relative à la création d'un dépositaire centrale et à l'institution d'un régime de l'inscription en compte de certaines valeurs.

${ }^{12}$ L'ouverture du capital de la Bourse de Casablanca à d'autres partenaires stratégiques internationaux est également envisagée. 
Statut (CFC) dont l'objectif est de favoriser le positionnement de la place financière marocaine en tant que place internationale et hub régional.

\subsubsection{Casablanca Finance City (CFC) comme hub régional et place financière internationale}

La dynamique des réformes du système financier marocain a été consacrée avec l'adoption de la loi n ${ }^{\circ} 44-10$ relative à la création de Casablanca Finance City $(\mathrm{CFC})^{13}$. Ce projet qui a démarré depuis 2010, a permis de faire de la place financière de Casablanca un hub économique et financier de dimension régionale et internationale. Le projet CFC permet de garantir aux investisseurs étrangers un environnement des affaires plus avantageux en matière juridique et fiscale.

Sur le plan institutionnel, le statut «CFC» est régi par la loi susmentionnée promulguée en juin 2014. La loi définit dans son article 11 les critères d'éligibilité au statut $\mathrm{CFC}^{14}$. Ce statut est accordé par la « commission $\mathrm{CFC}^{15}$ 》 aux entreprises financières (établissement de crédit, assurance et réassurance, sociétés de courtage en assurance et en réassurance, les sociétés financières de gestion d'actifs et les prestataires de services d'investissement) et aux entreprises non financières (les prestataires de services professionnels, les sièges régionaux et internationaux et les holdings). A fin Mars 2016, 104 statuts $\mathrm{CFC}$ ont été octroyés à 15 entreprises financières et à 89 entreprises non financières. Par pays, $80 \%$ des statuts $\mathrm{CFC}$ ont été attribués à 6 pays : Maroc (35), France (18), USA (13), Royaume Uni (7), Emirats arabes unis (5) et la Suisse (3).

Le statut $\mathrm{CFC}$ attribue aux entreprises labélisées CFC des avantages significatifs. En matière fiscale, les sociétés de services et les holdings bénéficient, au cours d'un exercice au titre de leur chiffre d'affaires à l'export et des plus-values mobilières nettes de sources étrangères, de l'exonération totale de l'IS pendant 5 ans consécutifs et de l'imposition au taux de 8,75\% au-delà de cette période. Les sièges régionaux, internationaux et les bureaux de représentation multinationales labélisés CFC bénéficient d'un taux de l'IS réduit de 10\%. En matière de l'IR, les salariés des sociétés CFC bénéficient au choix, soit du régime de droit commun, soit d'un taux spécifique de $20 \%$

${ }^{13}$ La loi n $44-10$ relative au statut de «Casablanca Finance city » telle que modifiée et complétée par la loi $n^{\circ} 68-12$. Promulgué en juin 2014.

${ }^{14} \mathrm{La}$ promotion institutionnelle du statut CFC est confiée à Casablanca Finance City Authority.

${ }^{15}$ La commission CFC est instituée par le Décret n²-11-323 du 7 chaoual 1432 (6 septembre 2011) pris pour l'application de la loi $\mathrm{n}^{\circ} 44-10$ au statut de «Casablanca Finance city »: Présidée par le Ministère chargés des finances et composée du directeur du trésor et des finances extérieurs, du directeur des assurances et de prévoyance sociale, un représentant de Bank Al-Maghreb, du directeur générale du CDVM (AMMC), du directeur et un représentant de Moroccan Financial Board. 
libératoire pendant 5 ans. En outre, les actes de constitution ou d'augmentation de capital sont exonérés pour toutes les sociétés $\mathrm{CFC}^{16}$. Le statut CFC permet aussi de faciliter les affaires : la création d'entreprise pendant $48 \mathrm{H}$, la facilitation du recrutement et l'obtention des titres de séjour pour les salariés étrangers et le visas business pour les professionnels.

Grace à ses avantages, aujourd'hui, ce projet est dans une phase de maturité. Il est caractérisé par intérêt croissant des investisseurs nationaux, régionaux et internationaux. En outre, le statut offre une multitude de services financiers dans des domaines des banques, de l'assurance, du conseil en investissement et de la gestion des actifs. Au fil de 6 années de son existence, Casablanca Finance City s'est positionnée en tant qu'une plateforme intégrée répondant aux besoins $\mathrm{du}$ continent en termes de financement et d'investissement. Selon le classement de l'indice de référence des centres financiers internationaux «Global Financial Centres Index -GFCI », CFC se positionne la $33^{\text {ème }}$ place à l'échelle mondiale parmi 86 places financières internationales (Ministère de l'Economie et des Finances, 2016).

Ce positionnement témoigne le progrès et le dynamisme du secteur financier marocain ce qui a promu des partenariats avec des grandes places financières, notamment Montréal, Londres, Paris, Luxembourg, Singapour et Ile de Maurice. Cela a permis à la place financière de Casablanca d'améliorer son attractivité à l'échelle mondiale et d'améliorer ses performances en termes du nombre des entreprises cotées, de la capitalisation boursière et du volume des activités sur les transactions sur les valeurs mobilières.

\subsubsection{Analyse de l'introduction en Bourse des Valeurs Mobilières de Casablanca}

L'introduction d'une entreprise à la bourse des valeurs mobilières de Casablanca peut se faire de deux manières. La première consiste à émettre des actions sur l'un des compartiments du marché des actions et la deuxième par l'émission des obligations cotées.

Pour l'introduction au marché des actions, les entreprises peuvent s'introduire soit sur le marché principal réservé aux grandes entreprises, sur le marché développement réservé aux entreprises de taille moyenne ou sur le marché croissance des entreprises en forte croissance. Une entreprise peut s'introduire sur l'un de ces marchés s'elle respecte deux catégories de condition: les conditions préalables et les conditions additionnelles. Le tableau suivant synthétise les conditions d'accès dans chaque type de marché des actions.

\footnotetext{
${ }^{16}$ Note $n^{\circ} 53 / 2013$ du 21 février 2013 du Ministère de l'économie et des finances et de la direction générale des impôts «relative à la clarification de certains avantages fiscaux accordés aux sociétés ayant le statut « Casablanca Finance City » et à leurs salariés.
} 
Tableau 2 : Les conditions d'introductions en Bourse de Casablanca

\begin{tabular}{|c|c|c|c|}
\hline \multicolumn{4}{|c|}{ Les conditions préalables } \\
\hline Profil des entreprises & $\begin{array}{l}\text { Marché Principal } \\
\text { Grandes entreprises }\end{array}$ & $\begin{array}{c}\text { Marché } \\
\text { Dér eloppement } \\
\text { Entreprises de } \\
\text { taille moyenne }\end{array}$ & $\begin{array}{c}\text { Marché Croissance } \\
\text { Entreprises en forte } \\
\text { croissance }\end{array}$ \\
\hline $\begin{array}{l}\text { Capitaux propres minimum en } \\
\text { millions de DH }\end{array}$ & $50 \mathrm{MDH}$ & Pas de limite fixée & Pas de limite fixée \\
\hline $\begin{array}{l}\text { Chiffre d'affaires minimum en } \\
\text { millions de DH }\end{array}$ & Pas de limite fixée & Plus de $50 \mathrm{MDH}$ & Pas de limite fixée \\
\hline Nombre d'exercices certifiés & 3 & 2 & 1 \\
\hline Comptes consolidés & $\begin{array}{l}\text { Oui (Si l'entreprise } \\
\text { dispose de filiales) }\end{array}$ & Facultatif & Facultatif \\
\hline \multicolumn{4}{|c|}{ Les conditions additionnelles } \\
\hline & Marché Principal & $\begin{array}{c}\text { Marché } \\
\text { Dér eloppement }\end{array}$ & Marché Croissance \\
\hline $\begin{array}{l}\text { Nombre de titres minimum à } \\
\text { émettre }\end{array}$ & 250000 actions & 100000 actions & 30000 actions \\
\hline $\begin{array}{l}\text { Montant minimum à émettre en } \\
\text { millions de } \mathrm{DH}\left({ }^{*}\right)\end{array}$ & $75 \mathrm{MDH}$ & $25 \mathrm{MDH}$ & $10 \mathrm{MDH}$ \\
\hline Convention d'animation & Facultative & 1 année & 3 années \\
\hline
\end{tabular}

Source : http://www.casablanca-bourse.com/bourseweb/content.aspx?IdLink=6\&Cat=5

Les conditions préalables sont liées au profil de l'entreprise alors que les conditions additionnelles sont liées aux transactions à effectuer après l'introduction effective au marché des actions. En plus de ses conditions qui semblent rigides, le processus d'introduction est relativement complexe et nécessite une expertise et un degré élevé de professionnalisme.

En dépit de toutes les réformes, la Bourse de Casablanca se caractérise par un faible nombre d'introduction. Elle compte 65 sociétés en 1993 et le solde des introductions et des sorties s'établie à 74 sociétés actuellement. Entre 2010 et 2017 la bourse a assisté à l'introduction de 10 sociétés $^{17}$. Le nombre des introductions et des sociétés cotées est relativement faible en comparaison avec d'autres pays de la région MENA (Tunisie, Egypte et la Jordanie).

\footnotetext{
${ }^{17}$ La liste et la date des sociétés introduites est disponible sur le lien : http://www.casablancabourse.com/bourseweb/Dernieres_Introductions.aspx . Ces sociétés sont : MSA (juin 2016), AFMA (décembre 2015), TMA (mais 2015), RDS (décembre 2014), TQM (décembre 2013), AFI (janvier 2012), S2M (décembre 2011), JET (décembre 2011), STR (juin 2011), SAH (novembre 2010).
} 
Tableau 3: Comparaison du nombre des sociétés cotées en bourse avec des pays émergents

\begin{tabular}{lccccccccccccccccc}
\hline Pays/Amnee & 2000 & 2001 & 2002 & 2003 & 2004 & 2005 & 2006 & 2007 & 2008 & 2009 & 2010 & 2011 & 2012 & 2013 & 2014 & 2015 & 2016 \\
\hline Introduites & 1 & 2 & 0 & 0 & 2 & 3 & 10 & 10 & 5 & 0 & 2 & 3 & 1 & 0 & 1 & 2 & 1 \\
Radiées & 2 & 0 & 0 & 3 & 1 & 2 & 1 & 0 & 1 & 1 & 4 & 1 & 0 & 1 & 2 & 2 & 1 \\
Cotées (Maroc) & 53 & 55 & 55 & 52 & 53 & 54 & 63 & 73 & 77 & 76 & 74 & 76 & 77 & 76 & 75 & 75 & 75 \\
Tunisie & 44 & 45 & 46 & 45 & 44 & 45 & 48 & 51 & 50 & 52 & 56 & 56 & 53 & 65 & 77 & 77 & 78 \\
Jordanie & 163 & 161 & 158 & 161 & 192 & 201 & 277 & 245 & 262 & 272 & 277 & 247 & 243 & 239 & 236 & 228 & 224 \\
Egypte & 1076 & 1110 & 1151 & 978 & 795 & 744 & 595 & 435 & 373 & 313 & 228 & 232 & 234 & 236 & 247 & 252 & 254
\end{tabular}

Source : Elaboré par nous-mêmes de la Bourse des valeurs mobilières de Casablanca et de la Fédération International des Bourses (WFE) ${ }^{18}$.

Le tableau ci-dessus nous montre que le nombre des sociétés marocaines cotées en bourse est de 77 à fin de 2016. Le marché principal compte 47 sociétés, le marché développement 16 sociétés et le marché croissance 12 sociétés. Cela nous montre que la majorité des sociétés cotées sont des grandes entreprises. Toutefois la migration d'un marché à un autre est possible sous le respect des conditions en vigueur. Le nombre des sociétés cotées au Maroc est relativement faible par rapport à la Tunisie (78 société cotées) mais il est très faible par rapport à l'Egypte qui compte 254 (1076 en 2000 et 1151 en 2002) et aussi en comparaison à la Bouse de Amman où le nombre des introductions n'a cessé d'augmenter depuis 2000 avec 163 sociétés, ce nombre atteint 224 sociétés à fin 2016. Par ailleurs, le tableau nous montre que la majorité des introductions en bourse de Casablanca ont été réalisées entre 2006 et 2008 avec l'entrée de 25 sociétés et la sortie de 2 sociétés. Le marché boursier marocain demeure marqué par la faiblesse des introductions des entreprises, surtout si l'on compare avec la situation de 1993 où le nombre des entreprises cotées était de 65 .

Selon une étude réalisée par la Bourse des Valeurs mobilières de Casablanca, il existe au Maroc 500 entreprises ayant les critères qu'exige une introduction en bourse, mais elles refusent de s'introduire. Les causes peuvent être classées en trois grandes catégories. D'ordre économique et financier : le financement de l'économie marocaine est dominé par le secteur bancaire, les entrepreneurs ont toujours une préférence pour les crédits bancaires afin d'éviter l'ouverture du capital et la multiplication des coûts (commission et fiscalité) d'introduction que sur les transactions (Rafiki, 2016) . D'ordre social et psychologique : la majorité des entreprises marocaines est de type familial, de ce fait, elles préfèrent l'approche patrimoniale à l'approche capitaliste (Abouch, 1998). D'ordre administratif: l'absence des efforts destinés à vulgariser l'approche finance de marché et ses avantages pour les entreprises, la lourdeur des procédures administratives en matière d'introduction en

${ }^{18}$ La Fédération international des bourses (Word Federation of Exchanges - WFE), site officiel : https://www.world-exchanges.org 
bourse, de financement et d'investissement, la transparence et la publication des informations financières qu'exige le fonctionnement du marché.

Pour l'introduction en Bourse via le marché obligataire, les conditions semblent moins contraignantes que celles du marché des actions. Le tableau suivant synthétise les conditions d'émission des emprunts obligataires.

Tableau 4 : Les conditions d'introductions au marché obligataires

\begin{tabular}{lc}
\hline Nombre d'exercices certifiés & 2 \\
\hline Comptes consolidés & Oui (si l'entreprise dispose de filiales) \\
Montant minimum à émettre en millions de DH & $20 \mathrm{MDH}$ \\
Maturité minimale & 2 années
\end{tabular}

Source : Bourse des valeurs mobilières (2017). Retired from http://www.casablancabourse.com/bourseweb/content. asp $x$ ? IdLink $=6 \&$ Cat $=5$

Afin de dynamiser le marché obligataire les conditions d'introduction ont été revues. Avant la dernière mise à jour de 2013, la valeur nominale d'une obligation cotée ne peut être inférieure à $10 \mathrm{dh}$ et celle des obligations non cotées ne peut être inférieure à $50 \mathrm{DH}$. Cette condition a été supprimée et seulement le montant d'émission est fixé à $20 \mathrm{MDH}$ pour une période qui ne peut être inférieure à 2 années (maturité).

Jusqu'au 30 Juin 2017, le nombre des entreprises opérationnelles dans le marché obligataire est de 48 des 75 cotées. Ces entreprises ont émis des obligations à taux révisables et sont au nombre de 5 et des obligations à taux fixes, soit 43 entreprises. Cependant, aucune entreprise n'a émis des obligations convertibles en action ou remboursables en action. Ainsi, 58\% des entreprises opérationnelles sont des banques ( 28 banques), les autres sont des sociétés de gestion de portefeuille et du secteur de bâtiment.

L'évolution des introductions en bourse affecte l'évolution des transactions, la performance du marché et le financement de l'économie. Nous analysons dans le point suivant la performance du marché boursier avant de montrer sa contribution au financement des PME marocaines.

\section{La performance de la bourse et financement de l'économie}

La performance du marché boursier est liée à la performance de ses principaux indicateurs du volume d'activité, de ses indices boursiers, de sa capitalisation boursière et son poids dans le PIB.

\subsection{L'analyse de l'évolution des transactions par catégorie}

Les transactions dans le marché boursier marocain, d'après l'analyse du tableau ci-dessous, se caractérisent par d'abord, l'importance en volume des opérations sur les actions que sur les obligations. Ce constat est imputé directement au faible nombre des entreprises opérationnelles sur le marché obligataire (48 sur 75 cotées). Ensuite, le volume des transactions sur le 
marché central est plus important que celui du marché des blocs. Cet écart est de plus en plus important sur le marché des actions que celui des obligations.

Le marché des actions est toujours marqué par la faiblesse des introductions et la rareté des opérations d'augmentation de capital. Alors que le marché des obligations est dominé à 99\% par les opérations d'émissions d'emprunt et les apports de titres sont quasiment nuls.

Tableau 5 : Evolution des volumes des transactions par catégorie (en millions de dirhams)

\begin{tabular}{|c|c|c|c|c|c|c|c|c|c|c|}
\hline & 2006 & 2007 & 2008 & 2009 & 2010 & 2011 & 2012 & 2013 & 2014 & 2015 \\
\hline \multicolumn{11}{|l|}{ Volume actions } \\
\hline 1. Marché Central & 58693 & 105992 & 78336 & 35943 & 51990 & 35211 & 29729 & 26450 & 27595 & 28758 \\
\hline 2. Marché de blocs & 9484 & 54721 & 22718 & 19439 & 29729 & 16089 & 12470 & 22101 & 11816 & 11751 \\
\hline Total $A=(1+2)$ & 68177 & 160713 & 101054 & 5382 & 81719 & 51300 & 42199 & 48551 & 39411 & 40509 \\
\hline 3. Introductions & 3603 & 6825 & 3851 & - & 837 & 416 & 27 & 1000 & 1127 & 893 \\
\hline 4. Apports de titres & 5174 & 6231 & 3083 & 3146 & 8484 & 33783 & 2063 & 644 & 2493 & 923 \\
\hline 5. Offres publiques & 9 & 796 & $\ldots$ & 113 & 10445 & 3 & 177 & 45 & 675 & 2408 \\
\hline 6. Transferts & 201 & 151 & 312 & 633 & 1156 & 978 & 617 & 1116 & 206 & 106 \\
\hline 7. Augmentations de capital & 1854 & 224 & 2883 & 3180 & 6231 & 7379 & 7469 & 3202 & 425 & 2045 \\
\hline Total $B=(3+4+5+6+7)$ & 10841 & 14227 & 10129 & 7072 & 27153 & 42559 & 10353 & 6007 & 4926 & 6375 \\
\hline I. T otal volume actions $(A+B)$ & 79018 & 174940 & 111183 & 62454 & 108872 & 93859 & 52552 & 54558 & 44337 & 46884 \\
\hline \multicolumn{11}{|l|}{ Volume Obligations } \\
\hline 8. Marché Centrale & 650 & 906 & 1202 & 3391 & 6314 & 4932 & 3205 & 2171 & 3355 & 2690 \\
\hline 9. Marché de blocs & 719 & 1443 & 6612 & 5201 & 3042 & 3665 & 4368 & 4713 & 1936 & 1706 \\
\hline Total $\mathrm{C}=(8+9)$ & 1369 & 2349 & 7814 & 8592 & 9356 & 8597 & 7573 & 6884 & 5291 & 4396 \\
\hline 10. Emissions & 2925 & 2675 & 3242 & 1482 & 1593 & 889 & 910 & 700 & 184 & 810 \\
\hline 11. Apports de titres & $\ldots$ & $\ldots$ & $\ldots$ & - & 109 & 23 & $\ldots$ & - & - & 1 \\
\hline Total D $=(10+11)$ & 2925 & 2675 & 3242 & 1482 & 1702 & 912 & 910 & 700 & 184 & 811 \\
\hline II. Total volume obligations (C+D) & 4294 & 5024 & 11056 & 10074 & 11058 & 9509 & 8483 & 7584 & 5475 & 5207 \\
\hline III. Totalgénéral(I+II) & 83312 & 179964 & 122239 & 72528 & 119930 & 103368 & 61035 & 62142 & 49812 & 52091 \\
\hline
\end{tabular}

Source : Elaboré par nous-mêmes à partir des rapports du (CDVM, 2010, 2013, 2015).

Il ressort ainsi du tableau que le volume global des transactions s'est établi à 52091 millions de dirhams à fin 2015 contre 179964 en 2007, soit une baisse de $71 \%$ et de $56,56 \%$ par rapport à 2010 . Cette baisse est continue depuis 2007 et elle est imputée à la progression négative des opérations sur le marché obligataire et à la faiblesse des opérations d'introduction. En outre, la baisse constatée entre 2007 et 2009 est plus importante mais aggravée sur le marché central des actions. Celle-ci était liée particulièrement à la crise financière où l'incertitude est le maître des transactions boursières.

Il faut noter également que la part des investisseurs marocains dans le volume global des achats représente $88 \%$ en 2015 contre $85 \%$ en 2014 et représente $82 \%$ et $83 \%$ dans le volume des ventes en 2015 et 2014 successivement (CDVM, 2015).

Globalement, la baisse continue du volume des transactions sur le marché boursier nécessite des investigations, notamment assez de réformes ont été introduites dans un environnement économique et politique stable. Le volume des transactions affecte directement la performance de la place financière et son attractivité à l'international.

\subsection{L'analyse de la performance du marché boursier}


L'analyse de la performance de la bourse des valeurs de Casablanca sur les 10 dernières années, nous montre une baisse structurelle de ses principaux indices à savoir MASI et MADEX. Le MASI a régressé de $-3,8 \%$ entre 2008 et 2015 contre $+22,2 \%$ entre 2000 et 2007 . Le MADEX a régresse de $-4 \%$ durant la période de 2008-2015 contre une performance positive de 23,4\% durant la période 2000-2007. La baisse terrible des indices sur les dix dernières années nous alerte à l'apparition d'une crise boursière, ce qui peut remettre en cause sa capacité à financer les investissements. Pour faire face à cette crise, le projet d'ouverture du capital de la bourse et les partenariats signés avec d'autres bourses sont les deux grandes solutions mises en place en 2015 et 2016.

Globalement, la financiarisation de l'économie d'un pays est apprécié par la capitalisation boursière et son poids dans le PIB $^{19}$. La capitalisation boursière renseigne sur la valorisation d'une place financière. Elle est déterminée par le total des capitalisations boursières de l'ensemble des entreprises cotées sur la place considérée.

Tableau 6 : L'évolution de la capitalisation boursière

\begin{tabular}{|c|c|c|c|c|c|c|c|c|c|}
\hline Indicateurs & \multicolumn{2}{|c|}{ Indices } & \multicolumn{3}{c|}{ Capitalisation boursière (CB) } & \multicolumn{3}{c|}{ Capitalisation Boursières par secteur (\%) } \\
\hline Année & MASI & MADEX & En Milliard Dhs & CB/ PIB (\%) & Banques & $\begin{array}{c}\text { Sociétés } \\
\text { financières }\end{array}$ & $\begin{array}{c}\text { Energies et } \\
\text { mines }\end{array}$ & Industrie & Services \\
\hline 2006 & 9479,45 & 7743,81 & 139,1 & 69,1 & 25,4 & 12,8 & 4,2 & 17,6 & 39,9 \\
\hline 2007 & 12694,97 & 10464,34 & 326,1 & 90,5 & 25,8 & 12,8 & 3,2 & 17,4 & 40,8 \\
\hline 2008 & 10984,29 & 9061,02 & 217,7 & 74,2 & 25,2 & 12,7 & 2,8 & 15,6 & 43,7 \\
\hline 2009 & 10443,81 & 8464,47 & 127,9 & 68 & 26,6 & 12,5 & 3,2 & 17,9 & 39,9 \\
\hline 2010 & 12655,20 & 10335,25 & 182,2 & 73,8 & 31,1 & 5,7 & 4,2 & 19,7 & 39,2 \\
\hline 2011 & 11027,65 & 9011,57 & 59,9 & 62,9 & 31,3 & 6,7 & 7,4 & 18,7 & 35,9 \\
\hline 2012 & 9359,19 & 7614,04 & 49,8 & 52,5 & 33,3 & 7,2 & 7,1 & 19 & 31 \\
\hline 2013 & 9114,14 & 7418,05 & 55,4 & 50 & 34,6 & 6,6 & 8,6 & 19,2 & 31 \\
\hline 2014 & 9620,11 & 7842,76 & 44,7 & 52,4 & 35,2 & 6,4 & 7,3 & 19,7 & 31,4 \\
\hline 2015 & 8925,71 & 7255,21 & 44,9 & 46,2 & 36,9 & 6,2 & 8,9 & 20,1 & 28 \\
\hline
\end{tabular}

Source : Elaboré par nous-mêmes du tableau de bord des indicateurs macro-économiques ( Direction des Etudes et des Prévisions Financières, 2016).

Il ressort du tableau ci-haut que, sur les dix dernières années, une diminution continue de la capitalisation boursière. Cette capitalisation est passée de 139,1 Milliards de dirhams en 2006 à 326,1 en 2007 pour se situer à 44,9 milliards de dirhams à fin 2015. La régression positive durant la période de 2006-2009 est imputée au nombre important des opérations d'introductions des entreprises ( 25 entreprises sur toute la période). Cependant, la chute terrible constatée en 2008 par rapport à 2009 est imputable à la crise financière internationale et la perte de la confiance des investisseurs en bourse. Globalement, entre 2008 et 2015 , la capitalisation boursière a reculé en moyenne de $-2,8 \%$ par an contrairement à la période de 2000-2007 où elle a

19 Financiarisation de l'économie par la bourse est appréciée par le ratio : Capitalisation boursière /PIB. 
progressé de 26,2\% en moyenne par an. En outre, le poids de la capitalisation boursière dans le PIB ne renseigne sur l'existence d'aucune tendance positive ou négative d'une année à l'autre. Toutefois, cet indicateur s'est amélioré en moyenne de 58,9\% durant la période 2008-2015 comparativement à la période 2000-2007 où son évolution atteint en moyenne 45,3\%.

Par ailleurs, les établissements bancaires et les sociétés financières accaparent les plus grandes parts de la capitalisation. Les banques accaparent en moyenne durant la période $2006-2015,30,5 \%$ de la capitalisation boursière. Sur la même période les sociétés financières représentent $8,96 \%$, les énergies et les mines $5,69 \%$, les industries $18,49 \%$ et les services $36,08 \%$.

De cette analyse, des conclusions sont tirées. La première conclusion est fondamentale, nous assistons à une dégradation fatale de l'ensemble des indicateurs boursiers (le volume d'activité, les indices et la capitalisation). La deuxième découle de la première et selon laquelle la bourse des valeurs mobilières de Casablanca semble déconnectée de l'économie marocaine et ne contribue que faiblement au financement des entreprises. Pour renforcer davantage son dynamisme, l'intégration des PME qui constituent plus de 95\% du tissu productif s'avère une nécessité. Pour ce faire, un programme appelé ELITE a été mis en place en partenariat avec London Stock Exchange Groupe. Dans le point suivant, nous analysons la contribution de la bourse des valeurs mobilières au financement des PME.

\section{Le cadre incitatif de financement des PME par le marché boursier}

La bourse des valeurs mobilières constitue une autre source de financement des PME marocaines. Cependant, tous les acteurs sont d'accord sur l'accès limité de ces entreprises au marché financier. Par ailleurs, ce constat est prouvé par le nombre de 12 PME cotées sur le marché croissance. Dans cette perspective, nous analysons la problématique de financement des PME marocaines par ce marché en deux points. Le premier s'articule autour les conditions d'accès au marché et le second concerne les mesures incitatives mise en place par les pouvoirs publics.

\subsection{Les conditions d'accès des PME à la bourse des valeurs de Casablanca}

Comme il a été démontré ci-haut, le financement direct des entreprises peut s'effectuer sur le marché monétaire élargi par l'émission des TCN ou sur le marché financier par l'émission des actions et des obligations.

Pour le marché monétaire, il y a trois instruments de financement regroupé sous forme des titres de créances négociables : les certificats des dépôts, les bons des sociétés de financements et les billets de trésorerie destinés à combler des besoins en trésorerie. Cependant, les PME comme toutes les entreprises financières ne peuvent être fiancées que par les billets de trésorerie. Pour ce faire, des conditions doivent être respectées, à savoir : au 
moins 5 ans d'existence, l'exigence de trois bilans certifiés, l'émission d'un montant minimal par billet de 250000 dirhams et la maturité allant de 10 jours à 1 an. En outre de ces conditions, des commissions sont exigées lors du placement auprès des OPCVM. On constate clairement que le marché des $\mathrm{TCN}$, particulièrement les conditions d'émission des billets de trésorerie sont contraignantes et destinées aux grandes entreprises. En outre, nous avons constaté au niveau de l'analyse de l'évolution des TCN que ce marché est dominé largement par les banques et les sociétés de financement ${ }^{20}$.

Pour le marché boursier, le troisième compartiment a été créé afin d'inciter les entreprises de taille moyenne et à forte croissance à s'introduire en bourse. L'accès au troisième compartiment, s'il n'exige aucune condition préalable en terme de capitaux propres et de chiffre d'affaires seulement un exercice certifié, les conditions additionnelles sont plus restrictives. Il s'agit d'émettre au moins 30000 actions de montant minimum de 10 millions de dirhams pour trois années d'animation. Cette dernière condition est absente au niveau du marché principal et de 2 ans sur le marché développement. Ces conditions semblent être moins incitatives à l'introduction en bourse en comparaison avec d'autres pays comme il montre le tableau suivant.

Tableau 7 : Comparaison des procédures d'admission des PME en bourse par pays

\begin{tabular}{c|c}
\hline Marché & Critères d'admission \\
\hline Altex (Johannesburg) & $10 \%$ du capital avec un minimum de 100 actionnaires. \\
\hline ACE Market (Malaysia) & $\begin{array}{c}\text { o du capital et au minimum 200 actionnaires détenant au } \\
\text { moins } 100 \text { actions chacun }\end{array}$ \\
\hline Marché alternatif (Tunis) & $\begin{array}{c}0 \% \text { du capital ou un minimum d'un million de dinars. } \\
\text { épartition des titres entre au moins } 10 \text { actionnaires ou } 5 \\
\text { institutionnels. }\end{array}$ \\
& $\begin{array}{c}\text { conditions de capital et de bénéfice ne sont pas exigées. } \\
\text { ssion possible pour les sociétés en cours de constitution par } \\
\text { appel public à l'épargne (cas de grands projets). }\end{array}$ \\
\hline Domestic Standard (Suisse) & $2 \%$ de l'offre répartie dans le public \\
\hline Source: Adapté par nous-mêmes du (CDVM, 2011).
\end{tabular}

Les conditions d'admission en bourse de ces pays (tableau ci-dessus) sont plus souples par rapport à ceux de la bourse de Casablanca. Ces marchés non réglementés offrent des conditions simplifiées et des avantages fiscaux aux PME qui ne disposent pas toujours des moyens financiers. L'objectif étant toujours de faciliter l'accès des PME au marché boursier, renforcer leur leurs fonds propres, améliorer le niveau de liquidité dans le marché, dynamiser le marché et améliorer sa lisibilité à l'échelle internationale.

Les commissions d'admission, de séjour et la fiscalité est un autre défi d'introduction des PME en bourse de Casablanca par émission d'actions. La commission d'admission est de $0,1 \%$ du capital social de l'exercice antérieur.

${ }^{20}$ Voir 1.1.2. Le marché des titres de créance négociables (TCN). 
Pour la commission de séjour annuelle, elle est payable par l'émetteur et dépond de la tranche du capital social (tableau suivant).

Tableau 8: La commission annuelle de séjour pour le marché des actions

\begin{tabular}{c|l|c}
\hline Tranche de capital social au 31/12/N-1 & é concernée & tant H.T/ versement annuel \\
\hline Inférieur à $100 \mathrm{MDH}$ & metteur & $5000 \mathrm{DH}$ \\
\hline De 100 à $200 \mathrm{MDH}$ & metteur & $10000 \mathrm{DH}$ \\
\hline Supérieur à $200 \mathrm{MDH}$ & metteur & $15000 \mathrm{DH}$ \\
\hline
\end{tabular}

Source : Bourse des valeurs mobilières. Retrieved from http://www.casablancabourse.com/bourseweb/Content.asp $x$ ?IdLink $=61 \&$ Cat $=5$

Ces commissions ont été fixées en 2013 et aucune mise à jour n'a été effectuée jusqu'à présent. Elles sont payables indépendamment des conditions d'évolution du marché et particulièrement du niveau de la structure de capital des entreprises cotées. En outre, ces commissions sont applicables aux trois compartiments du marché boursier : développement, croissance et principal, ce qui ne favorise par l'accès des PME au troisième compartiment. Par ailleurs, les commissions de négociation sont fixées à $0,1 \%$ H.T du montant des transactions sur les actions. Ces commissions sont assujetties à la TVA de $10 \%{ }^{21}$.

Pour le marché obligataire, il est ouvert à l'ensemble des sociétés anonymes (SA) sans conditions d'activités, de capital ou de bénéfice. Cependant, les conditions applicables n'envisagent pas des particularités ou des avantages pour les PME. De ce fait, ces entreprises restent marginalisées. D'ailleurs, nous avons constaté que ce marché est le moins dynamique puisqu'il ne contient que 48 entreprises, particulièrement des banques et des grands groupes du secteur immobilier. Selon le CDVM(2011), les PME marocaines n'ont pas toujours accès au marché obligataire, car les conditions sont hors de portée de cette catégorie d'entreprise.

Comme le marché des actions, l'admission au marché des obligations est soumise à une commission de cote de $0,005 \%$ H.T payable par le souscripteur et l'émetteur plafonnée à $10000 \mathrm{DH}$ H.T pour chacune des parties. En outre, la société émettrice doit payer une commission de séjour annuel une fois l'emprunt coté selon la grille tarifaire suivante :

Tableau 9: La commission annuelle de séjour pour le marché des obligations

\begin{tabular}{c|c|c}
\hline $\begin{array}{c}\text { Montant restant dû } \\
\text { au 31/12/N-1 }\end{array}$ & $\begin{array}{c}\text { Montant H.T } \\
\text { (Versement annuel) }\end{array}$ & $\begin{array}{c}\text { Plafond par } \\
\text { émetteur H.T }\end{array}$ \\
\hline Inférieur à $100 \mathrm{MDH}$ & $2500 \mathrm{DH}$ & $50000 \mathrm{DH}$ \\
\hline De 100 à $500 \mathrm{MDH}$ & $7500 \mathrm{DH}$ & $50000 \mathrm{DH}$ \\
\hline Supérieur à $500 \mathrm{MDH}$ & $15000 \mathrm{DH}$ & $50000 \mathrm{DH}$ \\
\hline
\end{tabular}

Source : Bourse des valeurs mobilières. Retrieved from http://www.casablancabourse.com/bourseweb/Content.aspx?IdLink=61\&Cat $=5$

${ }^{21}$ Conformément à l'article 101 de la Loi des finances n³5-05 pour l’année 2006. 
Les commissions annuelles de séjour dépendent du montant restant dû. De plus, le montant de versement annuel est soumis à une TVA de 10\% conformément à l'article 101 de la Loi des finances n³5-05 pour l'année 2006. Cependant, le plafond de la commission est plafonné à $50000 \mathrm{DH}$ pour l'ensemble des tranches du montant restant dû, ce qui constitue une contrainte pour l'émission des emprunts de faible montant.

Selon les autorités de la bourse, l'introduction en bourse nécessite un coût moyen global qui varie entre 2,2 et $5 \%$, ce qui constitue un frein à l'introduction des PME marocaines.

En conclusion, l'introduction des PME en bourse n'exige pas de conditions de capital ou de bénéfice ce qui constitue une opportunité pour celles qui veulent renforcer leurs fonds propres. Cependant, les exigences de transparence financière, de gouvernance et certification ne sont pas respectées par les PME marocaines. Ceci constitue certainement la première et la principale contrainte d'accès des PME en bourse. En outre, la multiplicité des grilles tarifaires (commissions et fiscalité) applicables dès l'introduction et sur l'ensemble des transactions expliquent la réticence des PME marocaines de s'introduire en bourse. Par ailleurs, la restructuration d'une entreprise s'avère nécessaire pour la conformité aux standards du marché, ce qui augmente les coûts d'introduction des PME. Dans le point suivant, nous mettons le point sur les mesures incitatives mises en place par les pouvoirs publics afin de faciliter l'accès des PME marocaines en bourse.

\subsection{Les mesures incitatives dédiées à l'introduction des PME en Bourse}

Les efforts et les réformes déployés par les pouvoirs publics (CGEM, ANPME, CCG et le CDVM) en partenariat avec les autorités de la bourse afin d'inciter les PME d'entrer en bourse ont affiché des résultats timides.

Le processus des réformes a commencé par la création du troisième compartiment en 2000. Après 17 ans d'existence ce compartiment ne compte que 12 entreprises.

Une autre incitation a été mise en place du $1^{\text {er }}$ juillet 2011 et valable jusqu'à décembre 2012 dédiée aux PME afin de les encourager à entrer en bourse $^{22}$. Cette offre consiste à octroyer aux PME un montant de 500000 dirhams au moment de l'introduction sous respect de certaines conditions. Ces conditions sont au nombre de 4 : avoir des capitaux propres inférieurs à 50 millions dirhams, s'introduire sur le compartiment Croissance ou Développement, émettre au minimum $20 \%$ du capital et s'introduire par augmentation de capital (avec ou sans cession). Suite à cette offre, 3

${ }^{22}$ L'offre est publiée sur la page suivante : La bourse des valeurs mobilières de Casablanca. (2011). Mesures incitatives pour encourager les PME à s'introduire en bourse. Retrieved from http://www.casablanca-

bourse.com/Bourseweb/DetailEvenement.aspx?IdArticle=309\&IdLangue=1 
entreprises ont été introduites en 2011 et une en 2012 contre la sortie d'une entreprise en 2011. Ces résultats mitigés montrent l'inefficacité de l'offre et l'incapacité des autorités à attirer les PME de s'introduire en bourse.

Dans le cadre du processus des réformes du marché financier à l'échelle nationale et régionale un partenariat a été signé en juin 2014 avec le London Stock Exchange Group (LSEG). La $1^{\text {ère }}$ concrétisation de ce partenariat est la mise en place d'un programme appelé ELITE ${ }^{23}$ destiné à préparer les PME aux exigences nécessaires afin de s'introduire en bourse. Ce programme mis en place au Royaume Uni en 2014, dans 24 pas Européennes en 2015 et adapté et déployé au Maroc par la Bourse de Casablanca, le $1^{\text {er }}$ pays non européen. En effet, ce programme, est né pour limiter l'écart ${ }^{24}$ entre les $^{1}$ exigences du marché boursier marocain et la réalité des entreprises marocaines notamment les PME (La bourse des valeurs mobilières de Casablanca, n.d.). Cela permet au marché marocain des capitaux d'introduire des entreprises répondant aux normes d'organisation, de gouvernance et de transparence qu'exige le fonctionnement de marché. Pour ce faire, une communié de 24 partenaires, composées des banques, des cabinets juridiques, des cabinets d'audit et d'expertise comptable, des cabinets de conseil financier, des fonds d'investissement, des organismes de formation été créée sous l'encadrement de la bourse de Casablanca.

Le programme de labélisation «ELITE » s'étale sur trois phases : se former, se préparer et se mettre en valeur.

$1^{\text {ère }}$ phase «Getready » : c'est la phase de formation des dirigeants et des actionnaires de l'entreprise qui participe au programme. La formation est répartie en 6 modules construits et adaptés selon les besoins des entreprises. Le but de cette étape est double, le premier est la préparation de l'intégration des changements culturels et organisationnels dans les entreprises éligible au programme, le deuxième est de permettre aux entreprises d'accéder au moyen de financement non nécessairement par l'introduction en boursier.

$2^{\text {ème }}$ phase «Get fit » : c'est la phase d'identification et d'évaluation des lacunes, d'une part, et de début d'introduction des changements sur le système organisationnel et financier de l'entreprise, d'autre part. Durant cette phase, la communauté des experts créée est à la disposition des entreprises éligibles afin de les conseiller et de les aider à introduire les changements, lever des fonds ou communiquer avec des investisseurs. Les principaux objectifs ainsi recherchés sont la consolidation de la pratique de la communication

${ }^{23}$ Le programme ELITE a été développé par le London Stock Exchange Group et déployé pour la $1^{\text {ere }}$ fois en 2012 par sa filiale BorsaItaliana en Italie.

${ }^{24}$ L'écart a été constaté lors des réunions de prospection avec les chefs d'entreprises conduite par la bourse des valeurs mobilière et une étude menée conjointement avec Maroc PME et l'APSB. 
financière et sociale, le développement du business plan de l'entreprise et la conception d'une stratégie de croissance pour l'entreprise ELITE.

$3^{\text {ème }}$ phase «Get value » : c'est la phase de mise en valeur où les entreprises labélisées «ELITE » accèdent à la plateforme digitale pour être lisible à la communauté financière, entrepreneuriale et aux entreprises ELITE au Maroc et à l'international. Ceci leur permet d'établir des collaborations, des partenariats avec des professionnels, des investisseurs, accéder à de nouvelle opportunités de financement : fonds d'investissement, émissions obligataires et s'introduire en bourse.

La plateforme comprend actuellement plus de 320 entreprises à l'échelle internationale, dont 6 ont été introduites en bourse en 2012. Pour l'état d'avancement de ce programme au Maroc, le lancement de la première cohorte en avril 2016 a permis l'intégration de 12 entreprises à ce programme $^{25}$. La deuxième cohorte a permis l'intégration de 12 entreprises $^{26}$. Malgré ces efforts déployés, le programme ne conduit pas automatiquement les PME ELITE à s'introduire en bourse. Pour plus d'efficacité à ce programme, il doit être normalement à la base de la création d'une bourse pour les PME marocaines.

Dans le même cadre des programmes d'aide de financement des PME, les pouvoirs publics ont mis en place des fonds dont la gestion est assurée par la Caisse Centrale des Garanties (CCG),

\section{Conclusion}

Les réformes et les efforts des pouvoirs publics destinés à inciter les PME à accéder aux marchés de capitaux demeurent vains. En effet, les PME marocaines ne disposent pas encore une bourse de valeurs mobilières. Ces entreprises n'ont la possibilité qu'à l'introduction au troisième compartiment (marché croissance). Malgré l'ensemble des efforts déployés par les pouvoirs publics depuis sa création, le nombre des introductions au troisième compartiment reste limité et ne contient actuellement que 12 entreprises. Comme les travaux antérieurs, les causes sont imputées aux facteurs institutionnels, financiers et fiscaux. Il s'agit notamment les conditions d'accès à chaque compartiment (croissance, développement et principal), d'une part, et les avantages financiers et fiscales à tirer après l'introduction en bourse, d'autre part. En effet, si les conditions d'introduction semblent moins

${ }^{25}$ Ces entreprises sont: KITEA, LAMACOM, 10 RAJEB, MAGHREB INDUSTRIES, ENERGY TRANSFO, MEDASYS, OUTSOURCIA, IWACO, DAMANDIS, DESTY TECHNOLOGIES, MUTRONIC, MANORBOIS

26 Ces entreprises sont: AGRO JUICE PROCESSING, AL KARAMA, BRICOMA HOLDING, BURINTEL, CMGP, COMPTOIR METALLURGIQUE MAROC, CUBAMAR/DECORA, GENERALE DE PROJETS, GHARB PAPIER ET CARTON (GPC), MADEC, PROFESSIONAL LABO, SOREMAR HOLDING. 
contraintes, les conditions d'émission des titres sont plus coûteuses et contraignantes surtout pour les PME.

En général, la Bourse des Valeurs de Casablanca, l'AMMC, la CGEM et l'ANPME s'accordent sur l'existence d'une distorsion entre les exigences du marché et le profil des PME marocaines. Pour réduire ce fossé, un programme d'envergure appelé ELITE a été lancé en 2014 pour aider et préparer les PME à fort potentiel de croissance de s'introduire en bourse. Malgré l'importance de ce programme, il n'oriente pas systématiquement les PME-ELITE vers l'introduction en bourse même s'elles répondent parfaitement aux conditions d'introduction en bourse. Pour plus d'efficacité à ce programme, il est vivement recommandé d'orienter sous certaines conditions les PME-ELITE vers l'introduction en bourse. Il peut également être comme point de départ de la création d'une bourse dédié aux PME. Cela peut faire l'objet d'une analyse empirique approfondie afin de déterminer ses retombées économiques et financières pour l'économie marocaine en général et pour les PME en particulier.

\section{References:}

1. Abouch. (1998). Système financier et développement: les conditions d'un financement auto-centré au Maroc. In A. du Colloque (Ed.), Pour une approche alternative à l'analyse du développement. Rabat: Actes du colloque.

2. BVM, C. (2012). Le marché boursier: structure et acteurs (Fiche thématique). Casablanca. Retrieved from www.casablancabourse.com

3. CDVM. (2010). Rapport annuel. CDVM. Casablanca. https://doi.org/10.1111/j.1365-2338.2011.02491.x

4. CDVM. (2011). Le financement des PME au Maroc. Maroc. Retrieved from www.CDVM.ma

5. CDVM. (2013). Rapport d'activité annuel. Casablanca.

6. CDVM. (2014). Rapport d'activité annuel. Casablanca.

7. CDVM. (2015). Rapport d'activité. Casablanca.

8. DEPF. (2016). Tableau de bord des indicateurs macro-économiques. Rabat.

9. La bourse des valeurs mobilières de Casablanca. (2012). Dossier de Presse: ELITE -Thinking Long Term. Casablanca. Retrieved from www.elite.com et www.casablanca-bourse.com

10. Levratto, N. (2009). Les PME définition, rôle économiques et politiques publiques. (De Boeck, Ed.) (1st ed.). Bruxelles.

11. Maroclear. (2015). Maroclear-Rapport annuel 2015. Casablanca. Retrieved from https://www.maroclear.com

12. Ministère de 1'Economie et des Finances. (2016). Revue AL-Maliya. 
$n^{\circ} 60$.

13. Rafiki, A. (2016). Contribution de la bourse et du système bancaire au financement des entreprises marocaines. Revue Marocaine Des Régimes Juridiques et Politiques, (10), 17-36.

14. Dahir $\mathrm{n}^{\circ} 1-96-246$ du 29 Chaabane 1417 (9 janvier 1997) portant promulgation de la loi $\mathrm{n}^{\circ} 35-96$ relative à la création d'un dépositaire centrale et à l'institution d'un régime de l'inscription en compte de certaines valeurs.

15. La loi $n^{\circ} 44-10$ relative au statut de «Casablanca Finance city » telle que modifiée et complétée par la loi nº6-12. Promulgué en juin 2014.

16. Note $n^{\circ} 53 / 2013$ du 21 février 2013 du Ministère de l'économie et des finances et de la direction générale des impôts « relative à la clarification de certains avantages fiscaux accordés aux sociétés ayant le statut « Casablanca Finance City » et à leurs salariés.

17. http://www.casablancabourse.com/bourseweb/content.aspx?IdLink=6\&Cat $=5$

18. http://www.casablancabourse.com/Bourseweb/DetailEvenement.aspx?IdArticle=309\&IdLan gue $=1$

19. La liste et la date des sociétés introduites en bourse : http://www.casablancabourse.com/bourseweb/Dernieres_Introductions.aspx.

20. La Fédération international des bourses (Word Federation of Exchanges - WFE), site officiel : https://www.world-exchanges.org 